Onkologe 2009 · 15:555-556

DOI 10.1007/s00761-009-1593-4

Online publiziert: 27. Mai 2009

(c) Springer Medizin Verlag 2009

\author{
U. Settmacher ${ }^{1} \cdot$ P.M. Schlag ${ }^{2}$ \\ ${ }^{1}$ Klinik für Allgemein-, Viszeral- und Gefäßchirurgie, Universitätsklinikum Jena \\ ${ }^{2}$ Comprehensive Cancer Center, Charité - Universitätsmedizin, Campus Mitte, Berlin
}

\title{
Transplantation und Onkologie
}

Das letzte Schwerpunktheft zum Thema Onkologie und Transplantation erschien vor nunmehr acht Jahren. Wir wollen mit der vorliegenden Ausgabe dieses spannende Feld für Sie aus klinisch relevanter Sicht neu aufarbeiten. Einige experimentelle Themen wie die Xenotransplantation sind bis heute noch nicht von Relevanz für die Praxis, sodass wir auf detaillierte aktuelle Erörterungen dazu verzichtet haben. Im Fokus stehen stattdessen aktuelle Indikationen zur Transplantation von Geweben und Organen bei Patienten mit systemischen und lokal umschriebenen Malignomen, das Potenzial der Malignomentstehung unter Immunsuppression nach Transplantation und die Nutzung von Immunsuppressiva mit antiproliferativer Wirkung, ebenfalls speziell nach Transplantation.

Allogene Stammzelltransplantationen werden bei Patienten mit akuten Leukämien und ungünstigem Risikoprofil in kurativer Intention durchgeführt. Der antineoplastische Effekt einer myelotoxischen bzw. myeloablativen Konditionierungstherapie wird hier mit einem immunologischen, antileukämischen Effekt nichteigener gewebeverträglicher Stammzellen kombiniert. Autologe Stammzelltransplantationen werden dagegen nach hochdosierter Radio-/Chemotherapie überwiegend bei fortgeschrittenen lymphatischen Neoplasien zur Verbesserung der Remissionsqualität eingesetzt. Die Hälfte aller autologen Transplantationen in Deutschland werden gegenwärtig bei $\mathrm{Pa}$ tienten mit multiplem Myelom nach Remissionsinduktion durchgeführt. Stammzellen können aus dem Knochenmark entnommen, nach medikamentöser Stimulation aus dem peripheren Blut gesammelt oder aus dem Nabelschnurrestblut asserviert werden. Dosisreduzierte Konditionierungen führen in der frühen Posttransplantationsphase zu geringerer Morbidität und Mortalität. Diese Entwicklung hat zu einer deutlichen Ausweitung der Indikation auf ältere Patienten bzw. auf Patienten mit Komorbiditäten geführt, die konventionelle myeloablative Konditionierungsprotokolle nicht oder nur mit erhöhtem Risiko tolerieren können.

Für die Transplantation solider Organe bleibt die Leber bis heute das einzige Organ, bei dem vorliegende primäre und auch ausgewählte sekundäre Malignome eine Indikation zum Organersatz im Sinne der radikalen Tumorentfernung als kurative Therapieoption darstellen. Patienten mit einem hepatozellulären Karzinom in einer zirrhotischen Leber profitieren von der Hepatektomie und der orthotopen Lebertransplantation. Patienten mit einer geringen Tumorlast haben nach Lebertransplantation die gleiche Langzeitprognose wie Patienten, die mit einer benignen Grunderkrankung transplantiert wurde. Dies und die Spenderorganknappheit hat zur Einführung der "Mailänder“ Kriterien für die Indikationsstellung in vielen Transplantationsregionen geführt. Auch mit der Einführung der Lebertransplantation mit Lebendspende konnte der Bedarf an Spenderorganen nicht gedeckt werden. Dieser Sachverhalt erhält bis zum heutigen Tag die Diskussion über prognoserelevante Kriterien der Indikationsstellung und Listung zur Lebertransplantation bei Patienten insbesondere mit einem fortgeschrittenen HCC aufrecht. Weitere aktuelle Schwerpunkte sind das Screening und die Frühdiagnostik des HCC bei Risikopatienten und das effiziente „Bridging“ 
während der Wartezeit der Patienten auf ein geeignetes Spenderorgan. An Studien zur Senkung der Rezidivrate nach Transplantation durch Modifikation der Immunsuppression und einer möglichen systemischen Chemotherapie wird derzeit gearbeitet. Auch bei anderen primären und sekundären malignen Tumoren der Leber kann die Lebertransplantation eine kurative Behandlungsoption für die betroffenen Patienten darstellen. Allerdings handelt es sich bei diesen Tumoren um eine sehr heterogene Gruppe, die erhebliche Unterschiede sowohl in Bezug auf die Tumoreigenschaften als auch die Behandlung und Prognose aufweist. Die Bedeutung der Lebertransplantation für das therapeutische Vorgehen bei diesen Tumoren ist daher bei den einzelnen Entitäten unterschiedlich, wobei auch die aktuellen Allokationskriterien den Einsatz der Transplantation beeinflussen und teilweise beschränken.

Einen weiteren Schwerpunkt in diesem Heft stellt das Auftreten maligner Tumorerkrankungen nach Transplantation dar. Hier werden große Anstrengungen in der Prophylaxe, Frühdiagnostik und Therapie unternommen. Durch die weiter verbesserten perioperativen Therapieregime auf allen Gebieten der Transplantationsmedizin rücken Komplikationen im Langzeitverlauf immer mehr in den klinischen Fokus. Die Entwicklung typischer maligner Tumoren unter Langzeitimmunsuppression ist Folge einer medikamentös herabgesetzten Immunantwort. Nach Transplantation solider Organe haben vor allem Hauttumoren, lymphoproliferative Erkrankungen und das Kaposi-Sarkom eine hohe Inzidenz. Es gibt experimentelle Belege dafür, dass Viren in Verbindung mit einer verringerten T-Zell-Abwehrleistung unter Immunsuppression eine bedeutende Rolle bei der Tumorgenese zukommt. Bei den Bemühungen, die Prädisposition transplantationsassoziierter $\mathrm{Tu}$ moren herabzusetzen und eine Verbesserung ihrer Behandlung zu erreichen, sind bereits deutliche Fortschritte erzielt worden.

Das Risiko der Tumorentstehung ist abhängig von der Intensität der Immunsuppression, von einzelnen Substanzklassen sowie deren Kombinationen. Neue Wirkstoffe, wie mTOR-Inhibitoren und Myco- phenolsäurederivate, vermögen über ihre antiproliferative Wirkung malignes Zellwachstum zu hemmen. In verschiedenen klinischen Studien konnte ein tumorsupprimierender Effekt der mTOR-Inhibitoren nachgewiesen werden. Für Mycophenolsäurepräparate liegen hauptsächlich experimentelle Daten vor, die eine günstige Beeinflussung von Lymphomen möglich erscheinen lassen. Generell muss festgestellt werden, dass es keine ausreichenden prospektiv, randomisiert und multizentrisch erarbeiteten Daten gibt, die eine evidenzbasierte Therapiestrategie beim präferenziellen Einsatz bestimmter Substanzgruppen oder Substanzkombinationen zur Reduktion des Tumorrisikos oder zur Therapie von Patientengruppen mit bereits initial erhöhtem Tumorrisiko gibt. Patienten mit erhöhter Wahrscheinlichkeit für eine Tumorerkrankung sollten, falls klinisch vertretbar, bevorzugt mit einem mTOR-Inhibitor immunsupprimiert werden. Bei Patienten mit manifester Erkrankung kann eine Umstellung der Immunsuppression den Verlauf ebenfalls günstig beeinflussen. Für Patienten mit einem Posttransplantationslymphom ist zusätzlich der Einsatz eines Mycophenolsäurederivates zu erwägen.

Wir wünschen Ihnen eine spannende und lehrreiche Lektüre dieses Schwerpunktheftes.

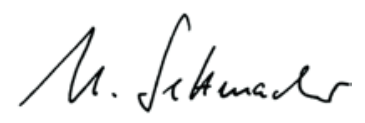

\section{U. Settmacher}

(Für die Herausgeber des Schwerpunktheftes)

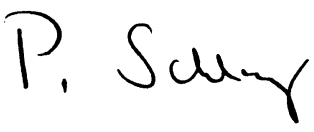

P. M. Schlag

(Für die Herausgeber)

\section{Korrespondenzadresse}

Prof. Dr. U. Settmacher

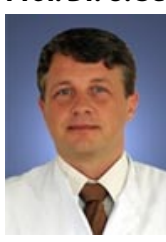

Klinik für Allgemein-, Viszeralund Gefäßchirurgie Universitätsklinikum Jena Erlanger Allee 101, 07747 Jena Utz.Settmacher@ med.uni-jena.de
Kongress-Digest: Überblick zu den internationalen Kongressen als PDF-Download

Anlässlich der großen internationalen onkologischen Kongresse bietet Ihnen das Internetportal der Deutschen Krebsgesellschaft e.V. ein neues Format der exklusiven Berichterstattung an. Ab sofort können Sie unter www.krebsgesellschaft.de verschiedene umfangreiche Artikel als PDF-Download erwerben. Im Mittelpunkt stehen die vier großen Tumorentitäten: Mammakarzinom, Bronchialkarzinom, Kolonkarzinom, Prostatakarzinom.

Sie erhalten eine ausführliche Übersicht über die wichtigsten Schwerpunktthemen, die interessantesten Daten und kontroverse Diskussionen. Die enge Zusammenarbeit mit anerkannten Experten, die selbst vor Ort waren, garantiert eine fundierte und auf wesentliche Daten und Fakten gestützte Berichterstattung. Gleichzeitig werden die Ergebnisse im Kontext bisheriger Standards erläutert und gewichtet. Auf diese Weise erhalten Sie schnell und übersichtlich eine Zusammenstellung und Interpretation der neuesten Forschungsergebnisse.

Zu den Kongressen der American Society of Clinical Oncology (ASCO) und der American Urological Association (AUA) finden Sie hier zu jeder der vier Krebsarten allgemeine Informationen (Diagnose, Früherkennung) und Informationen zur Therapie im frühen sowie fortgeschrittenen Krankheitsstadium.

Mehr Informationen unter www.krebsgesellschaft.de

Quelle: Deutsche Krebsgesellschaft e.V., Deutsche Krebsgesellschaft Internetportal 